\title{
Common and Unique Representations in pFC for Face and Place Attractiveness
}

\author{
Teresa K. Pegors, Joseph W. Kable, Anjan Chatterjee, and Russell A. Epstein
}

\begin{abstract}
Although previous neuroimaging research has identified overlapping correlates of subjective value across different reward types in the ventromedial pFC (vmPFC), it is not clear whether this "common currency" evaluative signal extends to the aesthetic domain. To examine this issue, we scanned human participants with fMRI while they made attractiveness judgments of faces and places - two stimulus categories that are associated with different underlying rewards, have very different visual properties, and are rarely compared with each other. We found overlapping signals for face and place attractiveness in the vmPFC, consistent with the idea that this region codes a signal for value that applies across disparate reward types and across both economic and
\end{abstract}

\section{INTRODUCTION}

Aesthetic evaluations, such as judging beauty and attractiveness, play an important role in our lives, affecting (for example) whom we choose to marry and where we would prefer to live. We can perform aesthetic evaluations on a wide variety of stimuli-a face can be beautiful or ugly, as can a house, room, or landscape. This suggests the possibility that a common evaluative signal might underlie different aesthetic judgments, analogous to the common signal that is believed to underlie different kinds of economic judgments. However, a counterargument is the fact that aesthetic judgments are rarely made across stimulus category ("Is this face more beautiful than this landscape?"). This makes them different from economic judgments, which usually involve comparison across disparate categories of goods, and suggests that a "common currency" for value might not be useful—or calculated—in the aesthetic domain.

Here, we test whether human $\mathrm{pFC}$ computes a common currency for value that is used for aesthetic judgments. Previous studies have shown that fMRI response in ventromedial pFC (vmPFC) during economic decision-making corresponds to the "utility" or "subjective value" of a stimulus (Bartra, McGuire, \& Kable, 2013). This response exhibits some degree of domain generality, insofar as it can be elicited by both money and consumer goods

University of Pennsylvania aesthetic judgments. However, we also identified a subregion of vmPFC within which activity patterns for face and place attractiveness were distinguishable, suggesting that some category-specific attractiveness information is retained in this region. Finally, we observed two separate functional regions in lateral OFC: one region that exhibited a category-unique response to face attractiveness and another region that responded strongly to faces but was insensitive to their value. Our results suggest that vmPFC supports a common mechanism for reward evaluation while also retaining a degree of category-specific information, whereas lateral OFC may be involved in basic reward processing that is specific to only some stimulus categories.
(Kim, Shimojo, \& O’Doherty, 2011; Levy \& Glimcher, 2011; Chib, Rangel, Shimojo, \& O’Doherty, 2009). However, the few studies that have attempted to compare noneconomic (e.g., social) rewards with monetary rewards have produced contradictory findings, sometimes showing overlapping activity in vmPFC and sometimes revealing nonoverlapping activity or no activity at all in this region (Lin, Adolphs, \& Rangel, 2012; Sescousse, Redouté, \& Dreher, 2010; Izuma, Saito, \& Sadato, 2008; Smith et al., 2004). It remains unclear whether a common evaluative signal exists that could apply to judgments very far removed from economic exchange such as aesthetic judgments.

To examine this issue, we scanned participants with fMRI while they made attractiveness judgments of faces and places. Our aim was to identify a common neural signal of value that operates across these two very different noneconomic stimulus classes. By using faces and places as stimuli, we intended to give our two categories the "best advantage" in terms of possible neural differences. Beautiful faces and beautiful places are associated with distinct reward outcomes: Beautiful faces offer the promise of reproductive success and social advancement (see Rhodes, 2006, for a review), whereas beautiful landscapes offer the promise of prospect/refuge, physical resources, and rest (White et al., 2010; Berman, Jonides, \& Kaplan, 2008; Appleton, 1975). Faces and places also differ substantially in their visual properties, to the extent that they are processed by distinct regions of visual cortex (Epstein, Harris, Stanley, \& Kanwisher, 1999; Kanwisher, Mcdermott, 
\& Chun, 1997). Face and place attractiveness are not typically assigned monetary values (although it is not impossible to assign monetary value to something without a market value; see Smith et al., 2004; Mitchell \& Carson, 1989). Therefore, a common response for face and place attractiveness is unlikely in the absence of a common currency-type, domain-general evaluative neural signal.

Previous work has shown that activity in vmPFC correlates with face attractiveness (Cloutier, Heatherton, Whalen, \& Kelley, 2008; Ishai, 2007; O'Doherty et al., 2003), although this is not always the case (Chatterjee, Thomas, Smith, \& Aguirre, 2009). A single study on the neural correlates of indoor place attractiveness showed no activity in vmPFC that correlated with parametric beauty ratings (Vartanian et al., 2013). Consequently, the question of whether face and place attractiveness are represented in the same or different brain regions remains open. To anticipate, our results suggest that there are both category-general and category-specific representations of attractiveness in vmPFC and a signal specific to face attractiveness in lateral OFC (latOFC).

\section{METHODS}

\section{Participants}

Thirty-four healthy, right-handed volunteers with normal or corrected-to-normal vision were recruited to participate in the fMRI study. All participants gave written informed consent according to procedures approved by the University of Pennsylvania Institutional Review Board. One participant was excluded because of an incidental finding, two participants were excluded because of technical issues with their anatomical images, and three participants were excluded because of excessive motion in the scanner. This left us with 28 participants whose functional data were analyzed ( 14 women, mean age $=$ 22.5 years).

\section{fMRI Acquisition}

Scans were performed at the Hospital of the University of Pennsylvania on a 3-T Siemens Trio scanner (Erlangen, Germany) equipped with a Siemens body coil and a 32-channel head coil. High-resolution T1-weighted anatomical images were acquired using a 3-D magnetizationprepared rapid gradient-echo pulse sequence (repetition time $=1620 \mathrm{msec}$, echo time $=3 \mathrm{msec}$, inversion time $=$ $950 \mathrm{msec}$, voxel size $=0.9766 \times 0.9766 \times 1 \mathrm{~mm}$, matrix size $=192 \times 256 \times 160$ ). T2*-weighted images sensitive to BOLD contrasts were acquired using a gradient-echo echo-planar pulse sequence (repetition time $=3000 \mathrm{msec}$, echo time $=30 \mathrm{msec}$, voxel size $=3 \times 3 \times 3 \mathrm{~mm}$, matrix size $=64 \times 64,46$ axial slices). The slices were tilted $-30^{\circ}$ from the AC-PC plane to reduce signal dropout (Deichmann, Gottfried, Hutton, \& Turner, 2003).

\section{Stimuli}

Stimuli were digitized $400 \times 400$ pixel color photographs of 144 places and 144 faces chosen to span a wide range of attractiveness. The places were natural environments with no manmade elements, spanning scene types such as swamps, mountain ranges, beaches, fields, and forests. The face set had equal numbers of men and women, and all faces were White, upright and forward-facing, with neutral-to-pleasant expressions, selected from the Glasgow Unfamiliar Face Database (homepages.abdn.ac.uk/m.burton/ pages/gfmt/Glasgow\%20Face\%20Recognition\%20Group. html), Radboud Database (Langner et al., 2010), the Center for Vital Longevity Face Database (Minear \& Park, 2004), CVL Face Database (Peter Peer, www.lrv.fri.uni-lj.si/facedb. html), Diana Theater Face Database (courtesy of Dr. Robert Schultz at the Center for Autism Research), and online searches. Face images were extracted from their original background, blurred slightly along the edges, cropped so that hair did not extend below the chin, and resized to span a height of 400 pixels. They were then placed on an abstract colored background created by phase-scrambling a single place image. This ensured that they subtended the same visual angle as the places while retaining a similar background color. A unique scrambling of the background image was used for each face (code used from visionscience.com/pipermail/visionlist/2007/002181.html).

These 288 stimuli were chosen from a larger set of 573 face and place images based on pilot testing intended to ensure that they covered a wide range of attractiveness, thus maximizing our ability to see neural activity related to this variability. In these pilot tests, 10 participants made Likert-scale ratings (1-8) of the visual attractiveness of each place and face. Images were blocked by category (face/ place), and participants used the keyboard to make ratings at their own pace, with instructions to spread their judgments across the whole 1-8 range. Each participant's ratings were then $z$-scored across all images, and these $z$ scores were averaged across participants for each image. The images were then divided into "low," "average," and "high" attractiveness bins according to whether they had a $z$ score below -0.5 (low), between -0.5 and 0.5 (average), or above 0.5 (high). Images were then chosen in equal numbers from these three attractiveness bins to make the final stimulus set.

\section{Design and Procedure}

The fMRI experiment consisted of six $4^{\prime} 57^{\prime \prime}$ scan runs, each of which was divided into two 36-sec face blocks, two 36-sec place blocks, and two 36-sec fixation (or "rest") blocks in which participants passively fixated on a central crosshair (Figure 1). Between each block was an additional $9 \mathrm{sec}$ of passive fixation. Eighteen seconds of fixation were added at the beginning and end of each run to allow the $\mathrm{T} 2 *$ signal to reach a steady state and to model the final hemodynamic response function 
Figure 1. Places and faces were presented to participants in blocks of 12 images. Two 36-sec fixation blocks were also included in each scan run. Each face appeared for $1 \mathrm{sec}$ with a 2-sec ISI. Participants made coarse attractiveness ratings for each image in the scanner ("low," "medium," and "high" attractiveness) and then rated the images again outside the scanner using a Likert scale of 1-8. The postrated images were presented to participants in one randomized block of faces and one randomized block of places (order counterbalanced across participants).

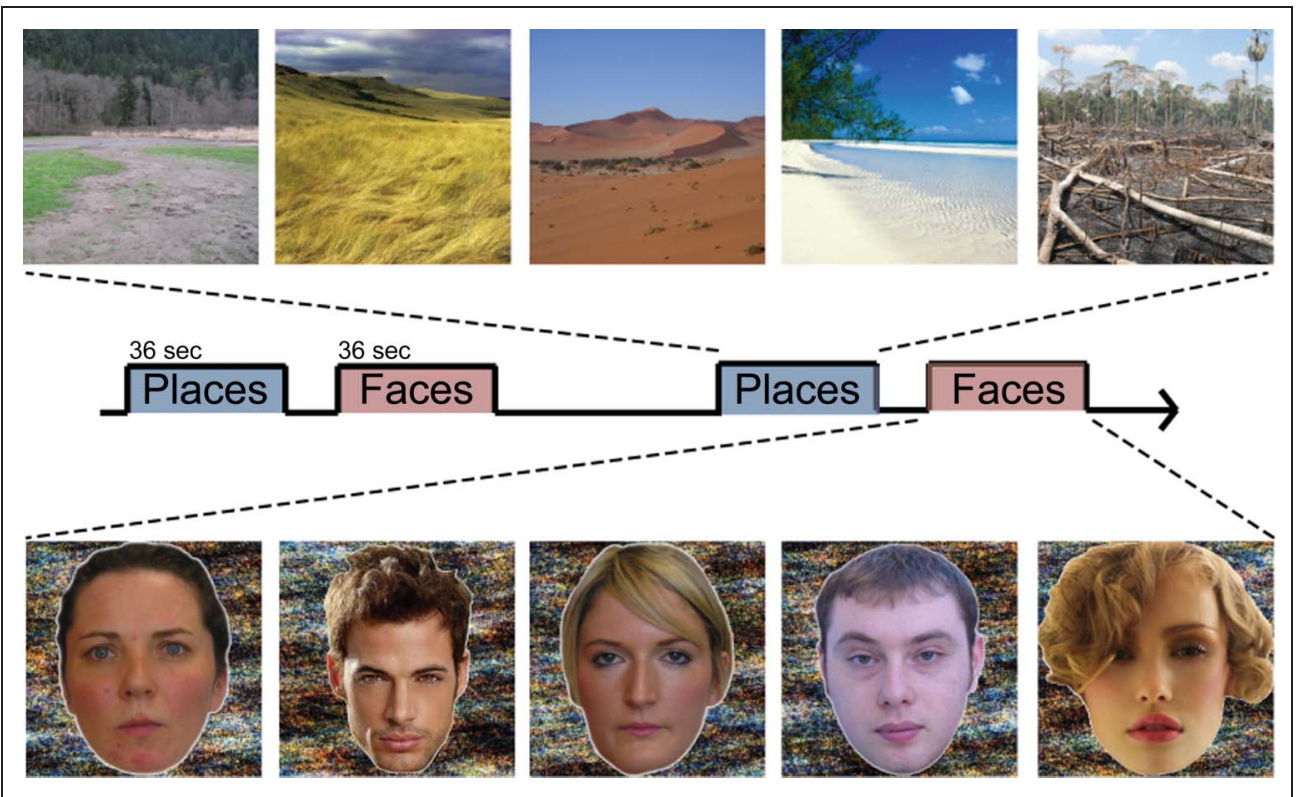

(HRF), respectively. During each face and place block, participants used a button box to give "low," "average," or "high" attractiveness ratings to 12 faces or places, each presented for 1 sec followed by a 2-sec ISI during which only a crosshair was on the screen. Participants made their ratings any time within the 3-sec trial; button assignment was counterbalanced across participants. Blocks were ordered such that no block type repeated twice in a row (including fixation blocks), and the block orders were counterbalanced across runs.

To acclimate participants to the distribution of attractiveness in the stimulus set, they were asked to rate before the scan the attractiveness of 24 faces and 24 places not used in the main experiment on a $1-8$ scale. These 48 images were chosen to span the entire range of attractiveness found in the main stimulus set. In addition, immediately after the scan session, participants were presented with the images from the main experiment again in a random order and asked to rate them on the same 1-8 scale. These postscan ratings were used in the subsequent fMRI analysis because they provided a finer-grained measure of face and place attractiveness than the high/average/low ratings made in the scanner.

Experiment trials within blocks were ordered such that the place and face attractiveness regressors used in the subsequent analyses would have maximal power to detect variability in fMRI response. Although these regressors were ultimately based on the attractiveness judgments made by the participants in the scanner, which could not be known in advance, we were able to obtain a rough estimate of their shape by using the high/average/low attractiveness ratings provided by the pilot participants. We generated 10,000 random orders of face and place stimuli that fit our experimental design, convolved the corresponding attractiveness ratings with a canonical HRF, and determined the power by calculating the ratio of variance before and after convolution. A "best" sequence of attractiveness levels was chosen (the one with the highest power), and images corresponding to these bins were drawn randomly from the stimulus set to create a unique stimulus sequence for each participant.

\section{fMRI Data Analysis}

Preprocessing and data analysis for individual participants were performed using the FMRIB Software Library (v.4.1.6; Jenkinson, Beckmann, Behrens, Woolrich, \& Smith, 2012). Functional images were corrected for differences in slice time acquisition and then de-obliqued to correct for the $30^{\circ}$ tilt slice acquisition. For each run, the first six volumes were removed to account for the fMRI signal not yet reaching steady state, and data were then motion corrected by spatially realigning each image with the central image in the run, registered to the subject-specific T1-weighted image using 6 degrees of freedom rigid-body transformations, and high-pass filtered to remove temporal frequencies below $0.0074 \mathrm{~Hz}$.

After preprocessing, we performed both univariate and multivariate pattern analyses on the data, as described below. An overview of the analysis scheme is provided in Figure 2.

\section{Univariate Analyses}

General linear modeling was used to estimate neural activity correlated with subject-specific ratings of face and place attractiveness. Each participant's postscan 1-8 ratings were used to create parametric regressors for face and place attractiveness, which extended across all six experimental scans. The model also included regressors corresponding to face and place in-scan RTs, as our 
Figure 2. Diagram illustrating the steps by which univariate and multivariate analyses were conducted. att. = attractiveness.
Step 1: Look for effects of place attractiveness in vmPFC using $n-1$ participants.

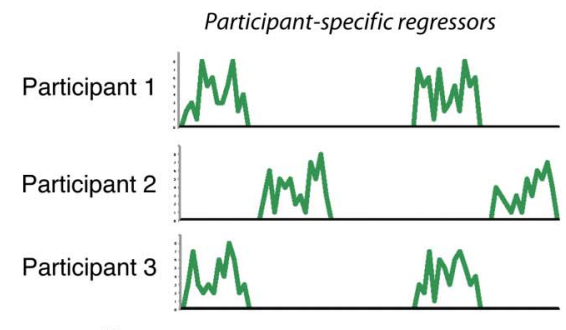

Perform small-volume cluster analysis

Participant $n-1$



Step 2: Use the resulting cluster from the group analysis to define an ROI.



Step 3: Use ROI to extract mean face and place attractiveness from left-out participant (participant $n$ ).

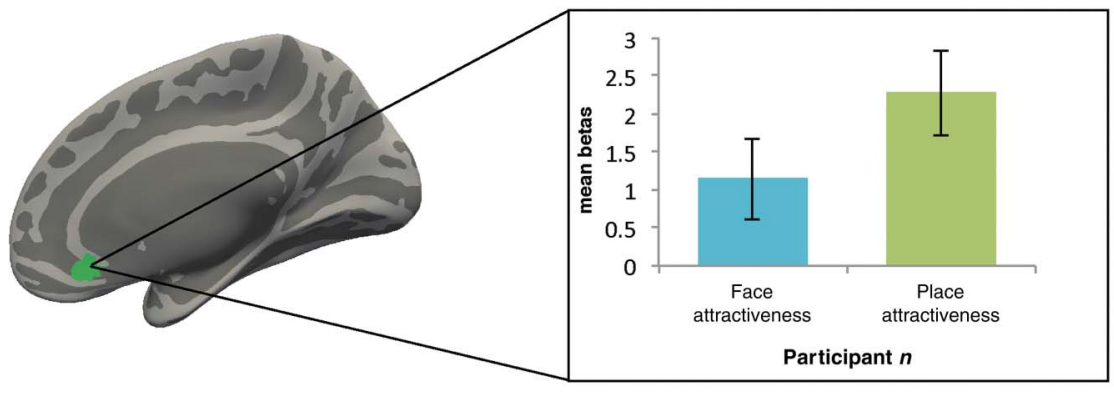

Step 4: Use ROI to perform pattern analysis from independent halves of left-out participant data.

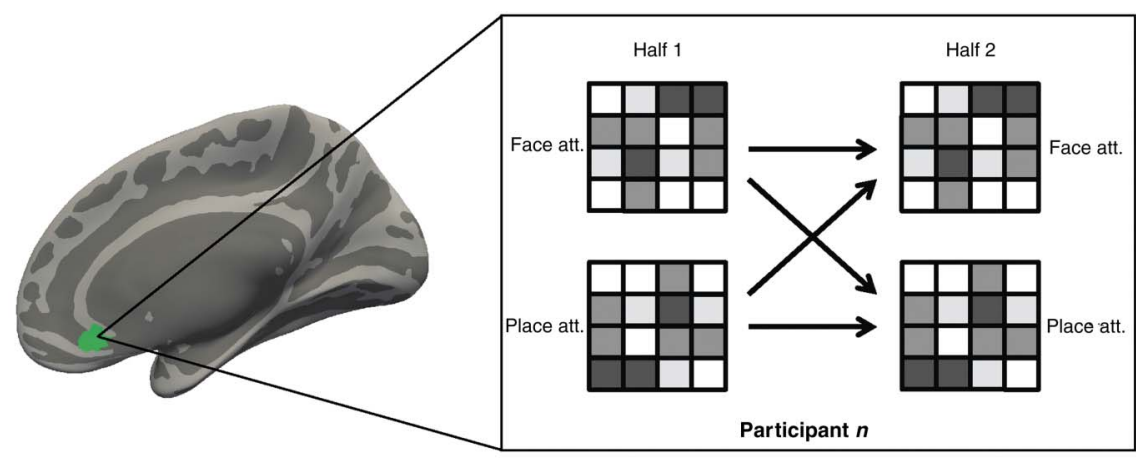

Step 5: Iterate steps 1-4, each with a different participant left out.

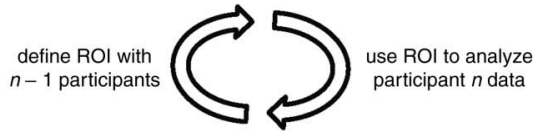

Step 6: Average results from Step 3 to obtain the univariate results, and average results from Step 4 to obtain the multivariate results. 
behavioral data revealed that RT was significantly negatively correlated with place attractiveness (but not face attractiveness). We did not include quadratic regressors for face and place attractiveness, in contrast to previous studies (e.g., Winston, O’Doherty, Kilner, Perrett, \& Dolan, 2007), because these quadratic regressors were strongly negatively correlated with RT. Finally, categorical regressors were added for face trials, place trials, and instruction screens, and nuisance regressors were added to account for between-scan variability and outliers (outliers calculated with the Gabrieli Lab's Artifact Detection Tools: www.nitrc. org/projects/artifact_detect/). All regressors, except scan indicators, were convolved with a canonical HRF.

We used this model to perform two sets of analyses. The first was a set of targeted analyses focusing on regions in the frontal lobe that have been implicated in the processing of information about stimulus value. The second was a whole-brain random effects analysis intended to find areas responding to face and place attractiveness without any a priori hypotheses about where these areas would be. For the targeted frontal lobe analyses, unsmoothed parameter estimates from the contrasts of interest were registered to the cortical surface using surface templates derived from each participant's T1-weighted anatomical image using Freesurfer's segmentation function (reconall). The data were then averaged across participants by spherically registering these participant-specific surfaces to the group brain (an average of the participant-specific surfaces, where vertex coordinates are calculated as the average Talairach coordinates of that vertex across participants). During this registration process, the data were smoothed on the group-average surface at 3-mm FWHM. Using the unfolded cortical surface for interparticipant registration in this manner reduces the variability when averaging across volumetric data associated with regions containing highly variable cortical folding patterns, which was especially important in this case because OFC is known to have a high degree of anatomical variability between participants (Chiavaras, LeGoualher, Evans, \& Petrides, 2001; Chiavaras \& Petrides, 2000). Random effects analyses were then performed on the contrasts of interest to identify regions within vmPFC and latOFC that responded to face and place attractiveness. Output was cluster-corrected for small volumes in vmPFC and latOFC and Bonferroni corrected to account for observations across two hemispheres (clusters defined at $p<.05$, uncorrected, and then permutation corrected to $p<.05$ using Freesurfer's simulation function to estimate the distribution of maximum cluster sizes under the null hypothesis). The medial surface a priori small volume was defined using a functional mask for vmPFC (Bartra et al., 2013), which we translated into surface space. As there is some evidence of categoryspecific signals in latOFC (e.g., Sescousse et al., 2010), we also used this area as an a priori small volume, defined by using the ventral surface of the "lateral orbitofrontal" ROI from Freesurfer's APARC library (taken from the DesikanKilliany atlas).
We performed a leave-one-subject-out iterative crossvalidation analysis (Kriegeskorte, Simmons, Bellgowan, \& Baker, 2009, supplementary discussion) on the significant clusters resulting from the targeted frontal lobe analysis to test whether activity within any clusters responding to face attractiveness or place attractiveness responded differentially to face versus place attractiveness. On each iteration, data from a single participant were held out as the test set. Clusters within vmPFC and latOFC responding to face or place attractiveness were then defined based on a group analysis of the $n-1$ remaining participants, using the procedures described above. The response in these clusters in the $n$th participant was then analyzed, and the procedure was repeated. This method gave us an estimate of the response in each cluster to face and place attractiveness, using independent data sets to define the boundary of the cluster and the strength of the effect.

For the whole-brain analyses, preprocessed data were smoothed with a 5-mm FWHM kernel, and parameter estimates for regressors of interest were obtained for each voxel for each participant. These were normalized to standard volumetric Montreal Neurological Institute 152 (MNI152) space using linear 12 degrees of freedom transformations, resampled to $2 \times 2 \times 2 \mathrm{~mm}$ voxels in this standard space, and submitted to group-level random effects analyses for contrasts of interest. The true Type 1 error rate for each contrast was calculated from FMRIB Software Library's randomise function using Monte Carlo simulations that permuted the signs of whole-brain data from individual participants $(10,000$ relabelings; method based on Freedman \& Lane, 1983). The resulting reported voxels are significant at $p<.05$, corrected for multiple comparisons across the whole brain.

We also performed a follow-up analysis, which examined response in functional ROIs in visual cortex. Because we did not conduct independent localizer scans for all participants, these ROIs were defined by using a set of 40 participants' localizer contrast files (19 came from our current study). These group-defined "parcels" were created using an algorithmic method, which is fully described in Julian, Fedorenko, Webster, and Kanwisher (2012). (We diverged from the Julian et al. description in that we chose a more liberal threshold of $p<.001$ for the face $>$ objects contrast maps.) Parcels were defined using the contrasts faces $>$ objects (fusiform face area, FFA), scenes $>$ objects (parahippocampal place area, PPA), objects $>$ scrambled objects (posterior lateral occipital cortex, LOC), and scrambled objects $>$ objects (early visual cortex, EVC). For the ROI analysis, we then translated individual participant contrast maps for face and place attractiveness into standard space and extracted parameter estimates for each participant within these group-defined ROIs.

\section{Pattern Analysis}

Our univariate analyses revealed clusters of activity in vmPFC that responded similarly to both face and place 
attractiveness. To further examine whether there were any differences between the signals for face and place attractiveness in vmPFC, we used a pattern classification analysis to test whether patterns of response rather than overall mean response within these clusters might reflect category-specific attractiveness information. Using wellestablished methods (Haxby et al., 2001), we split the data into independent halves (each consisting of three of the six scan runs), identified activity patterns for face and place attractiveness in each half, and then compared these patterns across halves to establish their reliability and distinguishability. Activity patterns in each half were calculated based on the same general linear model described above, except that the regressors spanned three scans instead of six. The resulting unsmoothed maps were then registered to the group-defined surface space, which allowed us to perform the classification analysis within the same independently defined, leaveone-subject-out vmPFC clusters that were used in our univariate analysis. Parameter estimates (beta values) were extracted for each participant's four independently defined vmPFC clusters, and classification was run iteratively over all possible split halves of the data (10 ways in which six scans can be split into two groups of three). Classification was considered successful if the average Pearson correlation between the face attractiveness patterns (or place attractiveness patterns) in opposite halves of the data (within-category comparison) was greater than the correlation between face and place attractiveness patterns in opposite halves of the data (cross-category comparison; Haxby et al., 2001). Raw correlation difference scores on which classification accuracy was based (e.g., face to face-face to place) were also calculated. Both the accuracy and correlation difference scores were then compared with chance (50\%) and zero, respectively, to determine if category-specific information was present in any of the vmPFC clusters.

\section{RESULTS}

\section{Behavioral Results}

Within-scan ratings of face and place attractiveness were strongly correlated with postscan ratings (Pearson's $r$ averaged across participants for faces $=.74, t(27)=$ 45.04, $p<.0001$ and for places $=.71, t(27)=35.17$, $p<.0001 ; p$ values reflect repeated-measures $t$ tests on correlation scores), and there was no significant difference between categories regarding the degree of correlation between these within-scan and postscan ratings (repeated-measures $t$ test on the difference between correlation scores: $t(27)=-1.4, p=.17)$. Postscan ratings for face and place attractiveness showed extremely high levels of consistency across participants (Cronbach's alpha: for faces $=.958$, places $=.956$ ). The within- and betweensubject consistency of attractiveness ratings confirms the validity of using the finer-scale postscan ratings to analyze the fMRI response.

Participant-specific means of face and place attractiveness ratings were not significantly different, although there was a trend for places to have higher mean ratings than faces $(t(27)=1.94, p=.06)$. Figure 3A displays histograms of postscan face and place attractiveness ratings. Postscan ratings for places were significantly negatively correlated with in-scan response times (Pearson's $r$ averaged across participants $=-.24$, repeated-measures $t$ test on correlation scores: $t(27)=-9.07, p<.0001)$. In contrast, there was no significant correlation between postscan face ratings and in-scan response times $(r=-.01, t(27)=-0.27, p=$ $.79)$. By plotting response time as a function of attractiveness, visual inspection revealed that, rather than a linear function, RT exhibited an inverted U-shaped relationship with both face and place attractiveness, peaking in the center range of attractiveness (Figure 3B). RTs for face judgments were highly symmetrical around the mean and therefore were not correlated with face attractiveness



Figure 3. (A) Average of participant-specific histograms of postscan attractiveness ratings. Error bars measure the standard error across participants. (B) RT plotted as a function of average attractiveness. Both face and place attractiveness exhibit an inverted U-shaped function, with the longest response times for midrange images. 
ratings, whereas RTs for place judgments had an elongated linear slope for the upper half of the attractiveness scale. For each item, we also calculated the mean RT and the standard deviation of rating judgments across participants. These measures were moderately correlated for both face and place stimuli (Pearson's $r$ : face stimuli $=.43$, place stimuli $=.57$ ). In other words, RTs were slower when there was greater disagreement among participants about the rating of an image, suggesting that RT may in part reflect the degree to which a participant is uncertain about their rating.

\section{Activity for Face and Place Attractiveness in Frontal Cortex}

We then turned to the primary question of interest: whether there was overlap between regions responding to face and place attractiveness in the frontal lobes. To answer this, we looked for effects of place and face attractiveness within frontal regions known a priori to exhibit subjective value signals: vmPFC and latOFC. We conducted the analyses in surface space to better account for the large variability in cortical folding patterns along the ventral surface of frontal cortex. We focused on two (nonoverlapping) regions.

Our vmPFC ROI was functionally defined based on Barta and colleagues' meta-analysis of subjective value responses in the brain (Bartra et al., 2013). Within this ROI, we observed clusters that responded linearly to face attractiveness at $p<.05$ (corrected for small volumes) in both hemispheres ("LvmPFC-face" and "RvmPFC-face"; see Table 1 and Figure 4) and clusters that responded linearly to place attractiveness in both hemispheres ("LvmPFCplace" and "RvmPFC-place"). Visual inspection makes clear that there is a great degree of overlap between the face and place clusters in each hemisphere. The face attractiveness clusters were found in similar locations to activity correlated with face attractiveness in previous studies (e.g., Cloutier et al., 2008; Ishai, 2007; O’Doherty et al., 2003; Figure 4).

To assess whether any of these clusters responded selectively to attractiveness for a single stimulus category, we performed a cross-validation analysis in which we iteratively defined the clusters on $n-1$ participants and then extracted parameter estimates for the "left-out" participant. This provides a stringent test of whether a cluster responded to both kinds of attractiveness: Although each cluster was defined based on its response to either face or place attractiveness, it was tested for its response to the other category in an independent data set. Repeatedmeasures $t$ tests comparing face with place attractiveness revealed that all vmPFC clusters showed a significant response to both face and place attractiveness (LvmPFCplace response to face attractiveness: $t(27)=2.11, p=$ .04 and response to place attractiveness: $t(27)=4.09$, $p=.0004$; LvmPFC-face response to face attractiveness: $t(27)=4.19, p=.0003$ and response to place attractiveness: $t(27)=3.70, p=.001$; RvmPFC-place response to face attractiveness: $t(27)=3.36, p=.002$ and response to place attractiveness: $t(27)=3.60, p=.001$; RvmPFCface response to face attractiveness: $t(27)=4.17, p=$ .0003 and response to place attractiveness: $t(27)=2.18$, $p=.04$; statistics obtained by $t$ tests comparing extracted mean parameter estimates against zero). Importantly, all clusters were equally sensitive to both face and place attractiveness regardless of how they were initially defined (face attractiveness $>$ place attractiveness: LvmPFC-face, $t(27)=0.09, p=.93 ;$ RvmPFC-face, $t(27)=1.04, p=$ .30; LvmPFC-place, $t(27)=-1.39, p=.18$; RvmPFC-place, $t(27)=-0.34, p=.73$; all $n s$, see Figure 4; all statistics were obtained by repeated-measures $t$ tests comparing extracted mean parameter estimates across categories.) These results suggest that vmPFC responds similarly to variation in attractiveness for these two categories, that

Table 1. Summary Table of Significant Clusters of Response for Face or Place Attractiveness within the vmPFC and latOFC ROIs (Contrast Shown in Italics)

\begin{tabular}{|c|c|c|c|c|c|c|c|}
\hline & & $m m^{2}$ & $x$ & $y$ & $z$ & Max Vertex Indices & Cluster-wise p \\
\hline \multicolumn{8}{|c|}{ Face attractiveness } \\
\hline vmPFC & $\mathrm{L}$ & 267.97 & -11.8 & 40.2 & -6.5 & 141127 & .016 \\
\hline vmPFC & $\mathrm{R}$ & 407.91 & 9.1 & 30.3 & -12.9 & 25109 & .000 \\
\hline latOFC & $\mathrm{R}$ & 131.09 & 32.0 & 33.8 & -7.7 & 93044 & .044 \\
\hline \multicolumn{8}{|c|}{ Place attractiveness } \\
\hline vmPFC & $\mathrm{L}$ & 588.35 & -5.6 & 33.3 & -0.2 & 83126 & .002 \\
\hline vmPFC & $\mathrm{R}$ & 145.59 & 9.2 & 30.7 & -10.0 & 57203 & .032 \\
\hline
\end{tabular}

Coordinates and vertex indices for peak values within the clusters are reported in fsaverage space (rather than our group-averaged space) for interpretability across studies. Cluster-wise $p$ values are permutation corrected for multiple comparisons within the vmPFC or latOFC and additionally Bonferroni corrected to account for observations across two hemispheres. $\mathrm{L}=$ left; $\mathrm{R}=$ right. 


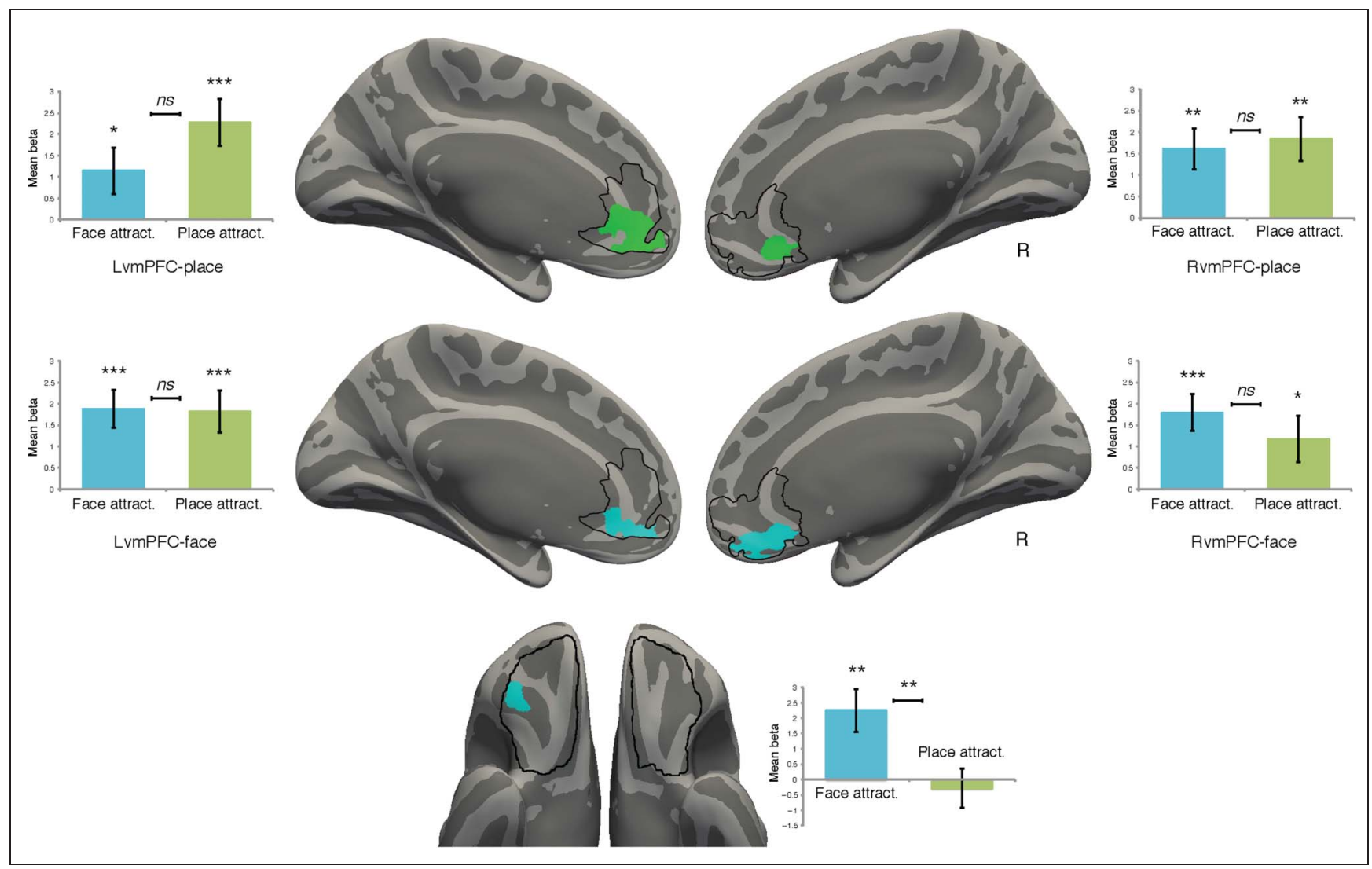

Figure 4. Cross-subject validation results for univariate cluster-corrected group analysis. Clusters responding to face attractiveness (blue) and place attractiveness (green) are shown on the cortical surface. Bar graphs show mean parameter estimates for face and place attractiveness within these clusters. These parameter values were extracted using a leave-one-subject-out cross-validation procedure so that data used to define the clusters were independent of data used to estimate the size of the effects and response patterns. The black outlines on the medial surface indicate boundaries of vmPFC, whereas black outlines on the orbital (i.e., ventral) surface indicate boundaries of latOFC. All vmPFC clusters show significant response to both face and place attractiveness. The face attractiveness cluster in the right latOFC only responds to face attractiveness. Attract. $=$ attractiveness.

is, clusters in vmPFC sensitive to face attractiveness are also sensitive to place attractiveness, and vice versa. Note that this is the case although the overall response in these regions was greater to faces than to places (faces $>$ places in LvmPFC-face: $t(27)=3.51, p=.002$; RvmPFC-face: $t(27)=4.52, p=.0001$; LvmPFC-place: $t(27)=4.44$, $p=.0001$; RvmPFC-place: $t(27)=3.18, p=.004)$.

Our second ROI was the latOFC. This region has been implicated in subjective value representation of distinct categories of reward (e.g., Sescousse et al., 2010), although value-based response is observed here less frequently than in vmPFC. Our analysis revealed a cluster in the right hemisphere whose response correlated with face attractiveness $(p<.05$ cluster-wise permutation corrected for small volumes; see Table 1 and Figure 4). We again used the cross-validation analysis to determine whether the attractiveness response in this cluster was category specific. A repeated-measures $t$ test found significantly higher response for face attractiveness compared with place attractiveness $(t(25)=2.64, p=.01)$. Indeed, place attractiveness response in the cluster was not significantly different from zero $(t$ test: $t(25)=-0.46, p=.65)$. Of note, the overall response to faces and places did not differ in this region $(t(25)=0.08, p=.94)$.

\section{Distributed Category-specific Encoding in vmPFC}

Our univariate analyses revealed that clusters in vmPFC that have significant mean response to one category of attractiveness also have a significant mean response to the other category and that the strength of these responses is not significantly different from each other. Although these results are in line with the "common currency" hypothesis for evaluative signals in vmPFC, it remains possible that these brain regions contain separate but intermixed valuation mechanisms for faces and places, which were not discriminable when responses were averaged over all voxels in the cluster. For example, Kahnt, Heinzle, Park, and Haynes (2010) showed that high and low rewards could be classified during reward anticipation, although there was no significant difference in mean response between the two conditions. We tested for a similar possibility by measuring vertex-wise activation patterns for face and place attractiveness in separate halves of the 
data within each vmPFC cluster and examining whether we could classify face versus place attractiveness across the split. Classification accuracy for the right hemisphere cluster defined by place attractiveness (RvmPFC-place) was above chance (accuracy $=58 \%, t(27)=2.63, p=.014$; see Figure 5). Accuracy in this cluster was similar for both categories, although only within-place accuracy was above the threshold for significance (face-face vs. face-place $=$ $58 \%, t(27)=2.5, p=.02$; place-place vs. face-place $=$ $58 \%, t(27)=2.0, p=.055)$. RvmPFC-face also showed a trend toward significant classification (accuracy $=55.8 \%$, $t(27)=1.9, p=.068)$ although neither individual category was significant on its own (face-face vs. face-place $=$ $55.9 \%, t(27)=1.66, p=.11$; face-face vs. face-place $=$ $55.7 \%, t(27)=1.55, p=.13)$. Given the fact that RvmPFC-face overlaps to a large degree with RvmPFC-place (see Figure 4) but also extends further anteriorly, these results suggest that the locus of category-specific information is centered within the RvmPFC-place. Accuracy was not significant for LvmPFC-place (accuracy $=50.3 \%, t(27)=0.1$, $p=.92$ ) or LvmPFC-face (accuracy $=46.1 \%, t(27)=-1.33$, $p=.20)$. We also examined the raw correlation scores themselves (upon which the accuracy measures were made) by calculating the difference scores between within- and cross-category Pearson correlations. These difference scores were significantly different from zero only within the same RvmPFC-place cluster that showed significant place versus face attractiveness classification (face/face - face/place difference score $=0.08, t(27)=2.08, p=.05$; place $/$ place face/place difference score $=0.08, t(27)=2.25, p=.03$ ).
To observe the focal point of pattern classification without using predefined clusters, we also performed a searchlight analysis, meaning that, for every vertex on the cortical surface, we centered a 7-mm-radius disc around that vertex and performed the same classification analysis. Within the vmPFC, peak correlation scores for both face and place attractiveness (face-face vs. face-place, place-place vs. face-place) were located close to the RvmPFC-place cluster but slightly more posterior (peak coordinates for face-face vs. face-place: 5.6, 19.4, -7.6; peak coordinates for placeplace vs. face-place: $5.7,17.3,-7.9$; analysis and coordinates are derived from fsaverage/MNI305 space). These searchlight results reinforce the finding that it is a ventral and posterior portion of vmPFC that contains categoryspecific information; the univariate-derived cluster appears to be picking up on the more anterior portion of this region.

\section{Whole-brain Analysis}

We next looked for regions whose response correlated with face attractiveness and place attractiveness outside our a priori regions in the frontal cortex. We observed bilateral response in the fusiform gyrus that was positively correlated with face attractiveness as well as a response in the right intraparietal sulcus (see Table 2). In contrast, no attractiveness-related activity for places survived wholebrain corrections, although we observed subthreshold activity in posterior cingulate, ventral striatum, vmPFC, and the region of parahippocampus gyrus/collateral
Figure 5. Multivertex pattern analysis of responses to face and place attractiveness in vmPFC. Bar graphs show classification accuracy when comparing response patterns across independent halves of the data. Accuracy (orange bars) was determined by comparing pattern similarity for attractiveness within a category with pattern similarity for attractiveness across categories. The breakdown by category (face-face vs. face-place and place-place vs. face-place) is also shown (blue and green bars). Only one cluster (RvmPFC-place) shows greater classification accuracy for same versus different attractiveness categories, although RvmPFC-face also shows a trend toward significance.

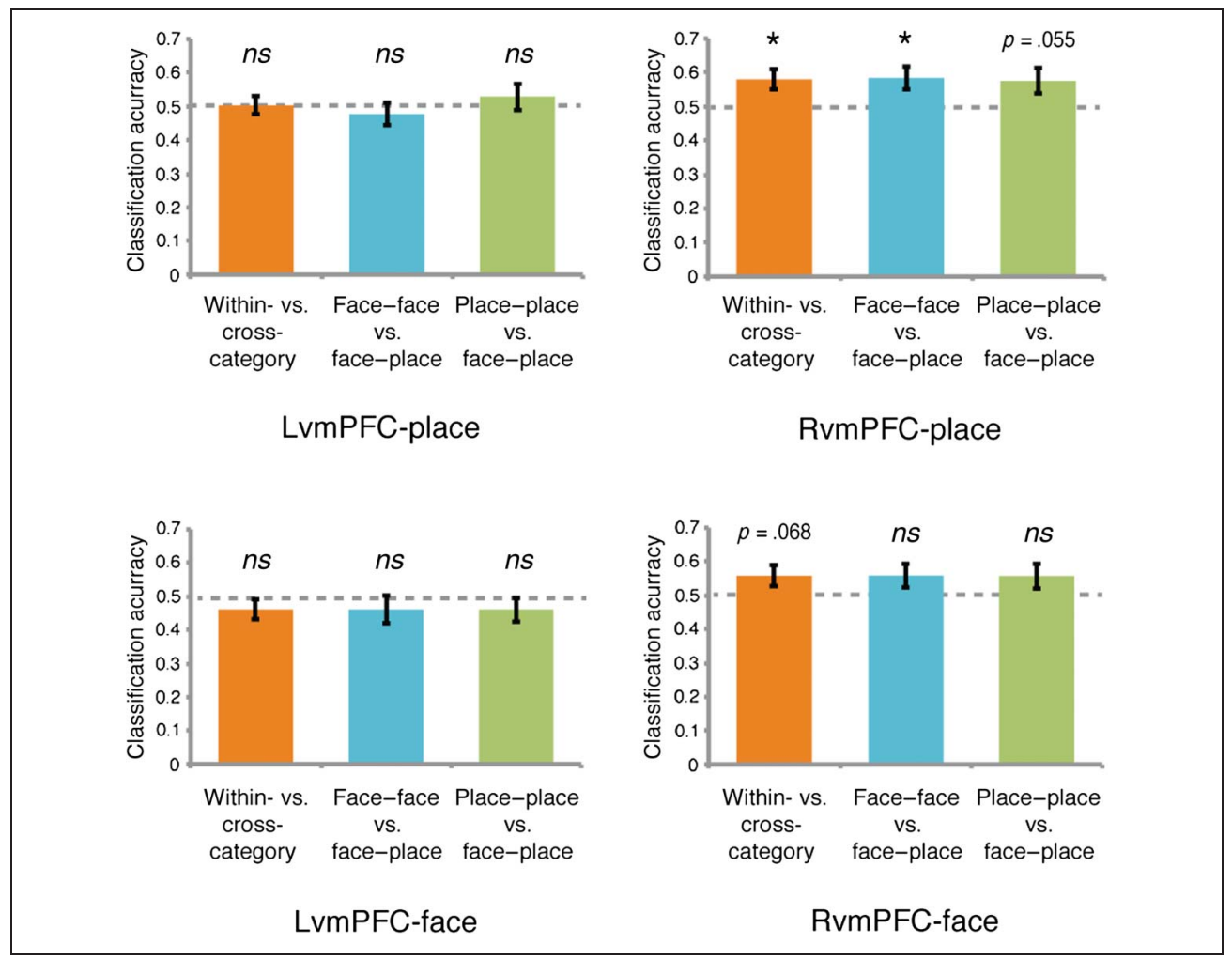


Table 2. Coordinates, Voxel Count, and Peak $t$ Values for Significant Clusters of Voxels

\begin{tabular}{|c|c|c|c|c|c|c|}
\hline & & Voxel Count & $x$ & $y$ & $z$ & Max $t$ \\
\hline \multicolumn{7}{|l|}{ Face $>$ place } \\
\hline Fusiform gyrus & $\mathrm{R}$ & 1135 & 42 & -74 & -12 & 11.03 \\
\hline FFA & $\mathrm{R}$ & a & 42 & -52 & -18 & 9.8 \\
\hline Occipital face area & $\mathrm{R}$ & a & 42 & -74 & -12 & 11.03 \\
\hline Cingulate sulcus & medial & 960 & 2 & 52 & 18 & 9.43 \\
\hline \multirow[t]{2}{*}{ Amygdala } & $\mathrm{L}$ & 921 & -20 & -8 & -16 & 11.03 \\
\hline & $\mathrm{R}$ & 710 & 20 & -8 & -14 & 11.7 \\
\hline Anterior cingulate gyrus & medial & 603 & 2 & 28 & -12 & 7.63 \\
\hline Posterior cingulate gyrus & medial & 404 & 4 & -52 & 20 & 8.62 \\
\hline Inferior occipital gyrus & $\mathrm{L}$ & 341 & -42 & -82 & -16 & 9.62 \\
\hline Inferior frontal sulcus & $\mathrm{R}$ & 251 & 48 & 8 & 20 & 7.06 \\
\hline Middle temporal gyrus & $\mathrm{R}$ & 127 & 58 & -4 & -26 & 7.46 \\
\hline Cerebellum & $\mathrm{L}$ & 67 & -32 & -82 & -38 & 6.36 \\
\hline Superior frontal gyrus & $\mathrm{R}$ & 66 & 20 & 38 & 50 & 6.7 \\
\hline \multirow[t]{2}{*}{ Supramarginal gyrus } & $\mathrm{L}$ & 51 & -58 & -64 & 26 & 5.98 \\
\hline & $\mathrm{R}$ & 37 & 56 & -60 & 24 & 7.19 \\
\hline Fusiform gyrus (FFA) & $\mathrm{L}$ & 46 & -40 & -50 & -24 & 6.93 \\
\hline Posterior orbital gyrus & & 27 & 38 & 32 & -16 & 6.04 \\
\hline \multicolumn{7}{|l|}{ Place $>$ face } \\
\hline \multirow[t]{2}{*}{ Temporal-occipital cortex } & $\mathrm{L}$ & 4415 & -26 & -50 & -10 & 21.4 \\
\hline & $\mathrm{R}$ & 4372 & 28 & -48 & -12 & 15.3 \\
\hline \multirow[t]{2}{*}{ Parahippocampal gyrus/collateral sulcus (PPA) } & $\mathrm{L}$ & a & -26 & -50 & -10 & 21.37 \\
\hline & $\mathrm{R}$ & a & 28 & -48 & -12 & 15.26 \\
\hline \multirow[t]{2}{*}{ Retrosplenial complex } & $\mathrm{L}$ & a & -14 & -56 & 10 & 10.01 \\
\hline & $\mathrm{R}$ & a & 14 & -52 & 8 & 10.35 \\
\hline \multirow[t]{2}{*}{ Middle occipital gyrus (occipital place area) } & $\mathrm{L}$ & a & -34 & -84 & 18 & 11.89 \\
\hline & $\mathrm{R}$ & a & 34 & -86 & 18 & 12.06 \\
\hline Lingual gyrus & $\mathrm{R}$ & 142 & 8 & -98 & -6 & 7.52 \\
\hline \multirow[t]{2}{*}{ Cingulate sulcus } & $\mathrm{R}$ & 81 & 4 & -44 & 50 & 8.19 \\
\hline & $\mathrm{L}$ & 66 & -10 & -36 & 44 & 6.55 \\
\hline \multirow[t]{2}{*}{ Cerebellum } & $\mathrm{L}$ & 25 & -14 & -46 & -52 & 6.67 \\
\hline & $\mathrm{R}$ & 17 & 16 & -46 & -52 & 6.3 \\
\hline \multicolumn{7}{|l|}{ Face attractiveness } \\
\hline Intraparietal sulcus (posterior) & $\mathrm{R}$ & 15 & 30 & -80 & 24 & 6.38 \\
\hline \multirow[t]{2}{*}{ Fusiform gyrus } & $\mathrm{L}$ & 9 & -42 & -54 & -16 & 6.09 \\
\hline & $\mathrm{R}$ & 4 & 22 & -54 & -14 & 5.8 \\
\hline Intraparietal sulcus (anterior) & $\mathrm{R}$ & 4 & 30 & -72 & 24 & 5.83 \\
\hline
\end{tabular}

Whole-brain maps for each contrast were thresholded at $p<.05$, permutation corrected for multiple comparisons. Coordinates are reported in MNI152 space. ${ }^{\mathrm{a}}$ Peaks are part of a larger cluster. 
Figure 6. Whole-brain maps for face (blue) and place (green) attractiveness displayed at $p<.0005$, uncorrected. The FFA is outlined in light blue, and the PPA is outlined in light green. Note that, at this more liberal threshold, vmPFC and ventral striatal activity is visible for place attractiveness, although these regions did not survive volumetric whole-brain corrections

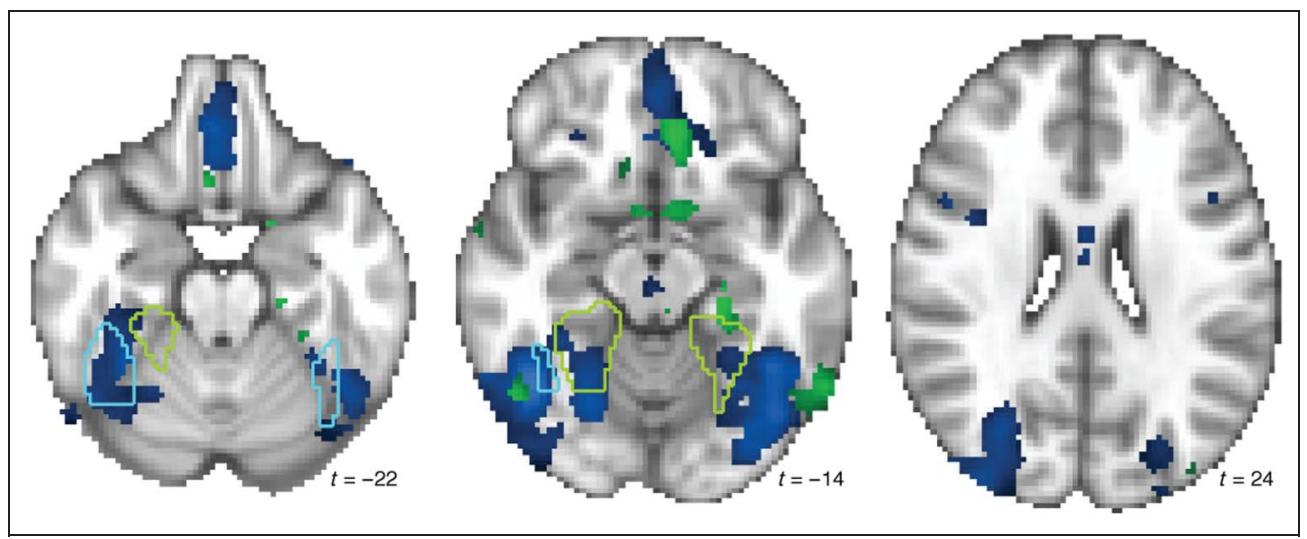

sulcus/hippocampus ( $p<.001$, uncorrected). In a direct contrast of face attractiveness versus place attractiveness, no voxels survived whole-brain corrections.

To explore whether the areas that responded to face and place attractiveness overlapped with face- and place-selective visual regions that have been identified in occipito-temporal cortex, we conducted an ROI analysis using independently defined ROIs for FFA, PPA, a posterior object-selective region (LOC), and EVC. Somewhat surprisingly, face attractiveness was positively correlated with activity in all higher level regions (right FFA: $t(27)=$ $2.9, p=.007$; left FFA: $t(27)=2.2, p=.037$; right PPA: $t(27)=3.8, p=.0008$; left PPA: $t(27)=2.5, p=.017$; right LOC: $t(27)=3.37, p=.002$; left LOC: $t(27)=3.05, p=$ $.005)$, whereas place attractiveness only showed positively correlated activity within right LOC $(t(27)=2.1, p=.04)$. Correlations between place attractiveness and fMRI response were nonsignificant for right FFA $(t(27)=0.8, p=$ $.41)$, left FFA $(t(27)=0.7, p=.46)$, right PPA $(t(27)=0.35$, $p=.73)$, and left PPA $(t(27)=0.00, p=.997)$. Neither face nor place attractiveness was significantly correlated with activity in EVC. Figure 6 shows the location of the functional ROIs and activity related to face and place attractiveness in visual regions.

Finally, for completeness, we compared categorical differences in activity between face and place trials (irre-

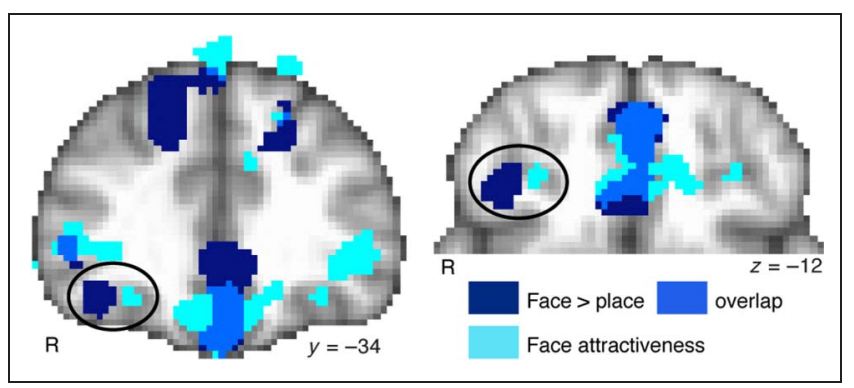

Figure 7. Relationship between face category effect and face attractiveness effect in PFC. The face $>$ place effect (dark blue) was thresholded at $t>4$.0, whereas the face attractiveness effect (light blue) was thresholded at $t>2.0$. Both contrasts were binarized, and the overlapping activity is displayed in pale blue. Peak response for the categorical effect is located lateral to peak response for face attractiveness in the right OFC (circled) spective of attractiveness). We observed significantly greater response during place compared with face trials in regions previously reported to respond preferentially to places and scenes (bilateral PPA, retrosplenial complex, occipital place area/transverse occipital sulcus). Conversely, we observed significantly greater response during face compared with place trials in visual regions previously reported to respond preferentially to faces (bilateral FFA and OFA) and also in posterior cingulate, bilateral amygdala, vmPFC, and surprisingly, a region of right latOFC. (Table 2 reports the MNI coordinates of all significant clusters for this comparison.) Because we had also observed activation in right latOFC for face attractiveness, we sought to determine whether the right latOFC region defined by our face $>$ place contrast also responded to face attractiveness. We defined this region as an ROI, thresholded at $t>3.5$ on the group map, and extracted the beta values from each participant's response to face attractiveness. Unexpectedly, this region's response was not significantly correlated with face attractiveness $(t(27)=0.75 p=.46)$. Figure 7 shows an overlay of both the face $>$ place map and the face attractiveness map, demonstrating that the face $>$ place peak response is located on the posterior orbital gyrus, whereas peak activity for face attractiveness is more medially located within the sulcus.

\section{DISCUSSION}

Attractive faces and attractive places promise very different rewards to a person, and comparisons are not often made between these rewards. Despite this, our results demonstrate that the vmPFC exhibits both category-general and category-specific responses to attractiveness. Clusters sensitive to face attractiveness in vmPFC were also sensitive to place attractiveness; however, a multivertex pattern analysis found that place and face attractiveness were distinguishable in one of these clusters, which was located in the posterior and ventral portions in the right hemisphere. These results suggest that some parts of vmPFC might encode category-general reward signals even when the stimuli are not exchangeable goods, whereas other parts might retain information about category-specific 
rewards. In the right latOFC, we observed two distinct face-specific regions: one sensitive to the categorical difference between faces and places but insensitive to face attractiveness and one sensitive to face (but not place) attractiveness but insensitive to categorical differences. As we found only face attractiveness and not place attractiveness signals in latOFC, this region may be more involved in basic reward processing that is specific to some but not all stimulus categories.

\section{Common Response to Face and Place Attractiveness in vmPFC}

In vmPFC, we identified clusters that were sensitive to face attractiveness and clusters that were sensitive to place attractiveness, and these face and place clusters were highly overlapping. Further analysis revealed a common response to face and place attractiveness: Clusters in vmPFC identified based on their response to face attractiveness responded equally strongly to place attractiveness, whereas clusters identified based on their response to place attractiveness responded equally strongly to face attractiveness.

These findings are consistent with a recent meta-analysis that demonstrated a region of vmPFC that encodes a common evaluative signal in studies where two or more categories were directly compared (Levy \& Glimcher, 2012). Although most of the studies in the meta-analysis involved economic and consumer goods, a few compared various social rewards to monetary rewards. Smith et al. (2004) showed overlapping activity in vmPFC for face attractiveness and monetary value when participants passively viewed intermixed images of faces and money. Lin et al. (2012) showed overlapping activity in vmPFC between monetary value and another type of social reward, pictures of smiling or angry people (paired with audio of emotionally matched words). Our results extend these findings by showing that, even in the case where both judgments are entirely outside the economic domain, in the realm of aesthetics, an overlapping evaluative signal exists in vmPFC.

Indeed, previous findings in the neuroaesthetics literature have suggested that vmPFC is generally involved in aesthetic evaluations (Ishizu \& Zeki, 2013; Brown, Gao, Tisdelle, Eickhoff, \& Liotti, 2011). For example, Ishizu and Zeki (2011) found overlapping response in vmPFC for beautiful paintings and music, demonstrating that a common evaluative mechanism in vmPFC is recruited by stimuli of different modalities (visual and auditory). However, it is possible that their participants evaluated all of the items within the same conceptual/reward framework of "artwork" because the items were presented as such. Our current results demonstrate an important extension to the results of Ishizu and Zeki, therefore, because faces and places in our study were not described as artwork but rather as distinct natural categories. Furthermore, they did not explore the possibility of separable distributed responses to visual and auditory artwork, whereas our pattern analysis revealed distinct face and place attractiveness patterns in at least one region that showed equal mean response between categories.

\section{Category-specific Attractiveness Coding in vmPFC}

Our pattern classification analysis revealed that a subregion in the right vmPFC (RvmPFC-place) contains separable distributed response patterns to face and place attractiveness, even while showing similar mean response. In contrast, the other three vmPFC clusters, which also showed significant mean response to both place and face attractiveness, did not display evidence of category-specific encoding, suggesting that category information in vmPFC may be restricted to a posterior and ventral subregion (see Figure 4; although null results in the other clusters cannot be used to ultimately reject the category-specificity hypothesis in those clusters).

Although our classification rates were not especially high, they were significantly greater than chance. Moreover, our results provide an intriguing parallel with results from a recent study by Mcnamee, Rangel, and O'Doherty (2013) who also used pattern classification to demonstrate category-specific encoding for food and trinket value in ventral vmPFC. They also demonstrated that dorsal regions of vmPFC showed both mean and distributed response patterns indicative of category-general encoding, a result that is consistent with our observation of overlapping mean activity for face and place attractiveness in vmPFC clusters, which did not exhibit category-specific responses in the classification analysis. Thus, our results provide independent support for the claim that vmPFC contains category-specific as well as category-general reward information, and furthermore, we show that these components exist for noneconomic rewards.

\section{Face-specific Activity in Right latOFC}

We observed two separate regions in the right latOFC that contained face-specific responses: one that exhibited a categorical preference for faces over places and one whose response scaled with face but not place attractiveness.

The previous literature on face attractiveness has not shown consistent results in latOFC. Two studies have found activity that positively correlated with face attractiveness in latOFC (left latOFC: Winston et al., 2007; right latOFC: Tsukiura \& Cabeza, 2011). In contrast, in a passive viewing task, Liang, Zebrowitz, and Zhang (2010) showed activity negatively correlated with face attractiveness in bilateral latOFC, although this study included disfigured faces, possibly causing an emotional/saliency signal to override a positive attractiveness response. O'Doherty et al. (2003) reported activity negatively correlated with attractiveness in right latOFC, but in their study, participants were making gender judgments rather than attractiveness judgments. When combined with these previous 
results, the current data suggest that positive latOFC activity for face attractiveness may only arise when participants are explicitly evaluating face attractiveness.

At least two other studies have reported a similar region of right latOFC that showed greater activation for faces than places (Von Der Heide, Skipper, \& Olson, 2013; Rajimehr, Young, \& Tootell, 2009). Interestingly, both groups used methods to reduce noise in this frontal region (surface-based group registration and a tilted acquisition, respectively). It is worth nothing that this latOFC region should be distinguished from the commonly observed face-selective region in the lateral inferior frontal cortex, a more dorsal region that is involved in social attention (see Nummenmaa \& Calder, 2009, for a meta-analysis).

No human neuroimaging study to our knowledge has shown a disjunction between regions exhibiting facespecific categorical response and regions exhibiting facespecific attractiveness response in latOFC. Our finding of this functional dissociation provides an important link to findings from the macaque, where multiple kinds of facespecific responses have been observed in OFC (Watson \& Platt, 2012; Tsao, Schweers, Moeller, \& Freiwald, 2008; Rolls, Critchley, Browning, \& Inoue, 2006; O Scalaidhe, Wilson, \& Goldman-Rakic, 1997). Using fMRI, Tsao et al. showed that, in macaque OFC, a patch on the orbital surface (lateral orbital sulcus) responded more strongly to faces with emotional expressions than to neutral faces, whereas a more lateral face-specific patch (inferior convexity) showed a categorical face response that did not vary with facial emotions. These anatomical locations are congruent with our own results: The categorical face patch was located on the posterior/lateral gyrus, and the face attractiveness patch was found in the lateral orbital sulcus. Although these similarities are suggestive of possible functional homologues, more research is needed to test the robustness and clarify the roles of these regions in humans, especially because the emotion patch in macaques responded to both positive and negative faces, whereas we were only able to test for linear responses to positive faces.

It is notable that we did not observe place-specific activity in latOFC. Places, unlike faces, may not act as a "basic" reward category in the same way as faces and food. The calculation of place beauty might instead be highly reliant on a dynamic process of integrating "component parts," such as spatial envelope or contrast/lighting, which may be associated with rewards only over time. Barron, Dolan, and Behrens (2013) have shown evidence that online construction of novel reward categories happens in vmPFC and hippocampus. In line with this theory, place attractiveness was found in our data to correlate with activity in vmPFC and at subthreshold levels in the hippocampal region.

\section{Visual Region Differences}

Consistent with previous findings (Chatterjee et al., 2009), we observed activity correlated with face attractive- ness across a large area of visual cortex, including faceresponsive (FFA) and object-responsive (LOC) regions. Importantly, this activity was not simply because of timeon-task, as RTs instead showed nonlinear patterns of response to attractiveness. Chatterjee et al. theorized that response to face attractiveness in the FFA reflects processing of face beauty per se, whereas response in LOC reflects processing of visual aesthetics regardless of the category. They hypothesized that place beauty might activate place-specific mechanisms in the PPA in addition to general visual aesthetic mechanisms in LOC. Although we did observe activity in right LOC correlating with place attractiveness, consistent with this prediction, we did not observe attractiveness-related activity in PPA for places. Rather, we observed an unexpected response to face attractiveness in this region.

Why might there be a weaker signal in visual cortex for place attractiveness? As discussed above, it may be the case that faces signify more immediate/basic rewards, and attractive faces may therefore recruit visual cortex as a part of an automatic approach response, whereas places would not. It could also be the case that we saw less activity for place attractiveness because the places spanned a greater variety of environment types (e.g., forests, beaches, deserts, fields) than did faces (males and females). For example, a recent study using a narrower range of place stimuli (indoor built environments) showed that place beauty was correlated with activity in the middle occipital gyrus, although there was no correlated activity within the ventral visual cortices (Vartanian et al., 2013). One study has reported greater activity in PPA for preferred scenes versus nonpreferred scenes (Yue, Vessel, \& Biederman, 2007), but it is important to note that this study markedly differs from our own in both task and stimuli, in that participants were asked to make ratings based on the content of the scenes, many of which were not places but images containing highly salient foreground objects, people, and animals. Although we did observe response to place attractiveness in the parahippocampal/hippocampal region anterior to the PPA, this did not survive the stringent threshold for significance in the whole-brain analysis. Taken as a whole with these previous findings, our data suggest that there are regions in the visual cortex that respond reliably to face attractiveness, but response to place attractiveness may depend on the nuances of the judgment task or the stimulus set.

\section{Conclusions}

Our data demonstrate a bridge between aesthetic and economic neural signals, in that a functionally similar evaluative mechanism in vmPFC is recruited for these disparate types of judgments. We expect that further research will continue to illuminate our understanding of this mechanism as well as the unique neural representations underlying specific reward categories. 


\section{Acknowledgments}

This work was supported by NIH grant EY-016464. T. Pegors was supported by the National Science Foundation Graduate Research Fellowship under grant no. DEG-0822. The authors would like to thank Omar Butt for his assistance with the surfacespace analyses.

Reprint requests should be sent to Teresa K. Pegors or Russell A. Epstein, Department of Psychology, 3720 Walnut Street, Philadelphia, PA 19104, or via e-mail: tpegors@sas.upenn.edu, epstein@psych.upenn.edu.

\section{REFERENCES}

Appleton, J. (1975). The experience of landscape. New York: Wiley.

Barron, H. C., Dolan, R. J., \& Behrens, T. E. J. (2013). Online evaluation of novel choices by simultaneous representation of multiple memories. Nature Neuroscience, 16, 1492-1498.

Bartra, O., McGuire, J. T., \& Kable, J. W. (2013). The valuation system: A coordinate-based meta-analysis of BOLD fMRI experiments examining neural correlates of subjective value. Neuroimage, 76, 412-427.

Berman, M. G., Jonides, J., \& Kaplan, S. (2008). The cognitive benefits of interacting with nature. Psychological Science, 19, 1207-1212.

Brown, S., Gao, X., Tisdelle, L., Eickhoff, S. B., \& Liotti, M. (2011). Naturalizing aesthetics: Brain areas for aesthetic appraisal across sensory modalities. Neuroimage, 5, 250-258.

Chatterjee, A., Thomas, A., Smith, S. E., \& Aguirre, G. K. (2009). The neural response to facial attractiveness. Neuropsychology, 23, 135-143.

Chiavaras, M. M., LeGoualher, G., Evans, A., \& Petrides, M. (2001). Three-dimensional probabilistic atlas of the human orbitofrontal sulci in standardized stereotaxic space. Neuroimage, 13, 479-496.

Chiavaras, M. M., \& Petrides, M. (2000). Orbitofrontal sulci of the human and macaque monkey brain. The Journal of Comparative Neurology, 422, 35-54.

Chib, V. S., Rangel, A., Shimojo, S., \& O'Doherty, J. P. (2009). Evidence for a common representation of decision values for dissimilar goods in human ventromedial prefrontal cortex. The Journal of Neuroscience, 29, 12315-12320.

Cloutier, J., Heatherton, T. F., Whalen, P. J., \& Kelley, W. M. (2008). Are attractive people rewarding? Sex differences in the neural substrates of facial attractiveness. Journal of Cognitive Neuroscience, 20, 941-951.

Deichmann, R., Gottfried, J., Hutton, C., \& Turner, R. (2003). Optimized EPI for fMRI studies of the orbitofrontal cortex. Neuroimage, 19, 430-441.

Epstein, R., Harris, A., Stanley, D., \& Kanwisher, N. (1999). The parahippocampal place area: Recognition, navigation, or encoding? Neuron, 23, 115-125.

Freedman, D., \& Lane, D. (1983). A nonstochastic interpretation of reported significance levels. Journal of Business E Economic Statistics, 1, 292-298.

Haxby, J. V., Gobbini, M. I., Furey, M. L., Ishai, A., Schouten, J. L., \& Pietrini, P. (2001). Distributed and overlapping representations of faces and objects in ventral temporal cortex. Science, 293, 2425-2430.

Ishai, A. (2007). Sex, beauty and the orbitofrontal cortex. International Journal of Psychophysiology, 63, 181-185.

Ishizu, T., \& Zeki, S. (2011). Toward a brain-based theory of beauty. PLoS One, 6, e21852.
Ishizu, T., \& Zeki, S. (2013). The brain's specialized systems for aesthetic and perceptual judgment. European Journal of Neuroscience, 37, 1413-1420.

Izuma, K., Saito, D. N., \& Sadato, N. (2008). Processing of social and monetary rewards in the human striatum. Neuron, 58 , 284-294.

Jenkinson, M., Beckmann, C. F., Behrens, T. E. J., Woolrich, M. W., \& Smith, S. M. (2012). FSL. Neuroimage, 62, 782-790.

Julian, J. B., Fedorenko, E., Webster, J., \& Kanwisher, N. (2012). An algorithmic method for functionally defining regions of interest in the ventral visual pathway. Neuroimage, 60 , 2357-2364.

Kahnt, T., Heinzle, J., Park, S. Q., \& Haynes, J.-D. (2010). The neural code of reward anticipation in human orbitofrontal cortex. Proceedings of the National Academy of Sciences, U.S.A., 107, 6010-6015.

Kanwisher, N., Mcdermott, J., \& Chun, M. M. (1997). The fusiform face area: A module in human extrastriate cortex specialized for face perception. Journal of Neuroscience, 17, 4302-4311.

Kim, H., Shimojo, S., \& O’Doherty, J. P. (2011). Overlapping responses for the expectation of juice and money rewards in human ventromedial prefrontal cortex. Cerebral Cortex, 21, 769-776.

Kriegeskorte, N., Simmons, W. K., Bellgowan, P. S. F., \& Baker, C. I. (2009). Circular analysis in systems neuroscience: The dangers of double dipping. Nature Neuroscience, $12,535-540$.

Langner, O., Dotsch, R., Bijlstra, G., Wigboldus, D. H. J., Hawk, S. T., \& van Knippenberg, A. (2010). Presentation and validation of the Radboud Faces Database. Cognition E Emotion, 24, 1377-1388.

Levy, D. J., \& Glimcher, P. W. (2011). Comparing apples and oranges: Using reward-specific and reward-general subjective value representation in the brain. Journal of Neuroscience, 31, 14693-14707.

Levy, D. J., \& Glimcher, P. W. (2012). The root of all value: A neural common currency for choice. Current Opinion in Neurobiology, 22, 1027-1038.

Liang, X., Zebrowitz, L., \& Zhang, Y. (2010). Neural activation in the "reward circuit" shows a nonlinear response to facial attractiveness. Social Neuroscience, 5, 320-334.

Lin, A., Adolphs, R., \& Rangel, A. (2012). Social and monetary reward learning engage overlapping neural substrates. Social Cognitive and Affective Neuroscience, 7, 274-281.

Mcnamee, D., Rangel, A., \& O’Doherty, J. P. (2013). Category-dependent and category-independent goal-value codes in human ventromedial prefrontal cortex. Nature Neuroscience, 16, 479-485.

Minear, M., \& Park, D. C. (2004). A lifespan database of adult facial stimuli. Behavior Research Methods, Instruments, \& Computers: A Journal of the Psychonomic Society, Inc, 36, 630-633.

Mitchell, R., \& Carson, R. (1989). Using surveys to value public good: The contingent valuation method. New York: Resources for the Future.

Nummenmaa, L., \& Calder, A. J. (2009). Neural mechanisms of social attention. Trends in Cognitive Sciences, 13, 135-143.

O Scalaidhe, S. P., Wilson, F. A. W., \& Goldman-Rakic, P. S. (1997). Areal segregation of face-processing neurons in prefrontal cortex. Science, 278, 1135-1138.

O’Doherty, J., Winston, J., Critchley, H., Perrett, D., Burt, D. M., \& Dolan, R. J. (2003). Beauty in a smile: The role of medial orbitofrontal cortex in facial attractiveness. Neuropsychologia, 41, 147-155. 
Rajimehr, R., Young, J. C., \& Tootell, R. B. H. (2009). An anterior temporal face patch in human cortex, predicted by macaque maps. Proceedings of the National Academy of Sciences, U.S.A., 106, 1995-2000.

Rhodes, G. (2006). The evolutionary psychology of facial beauty. Annual Review of Psychology, 57, 199-226.

Rolls, E. T., Critchley, H. D., Browning, A. S., \& Inoue, K. (2006). Face-selective and auditory neurons in the primate orbitofrontal cortex. Experimental Brain Research, 170, $74-87$.

Sescousse, G., Redouté, J., \& Dreher, J.-C. (2010). The architecture of reward value coding in the human orbitofrontal cortex. The Journal of Neuroscience, 30, 13095-13104.

Smith, S. M., Jenkinson, M., Woolrich, M. W., Beckmann, C. F., Behrens, T. E. J., Johansen-Berg, H., et al. (2004). Advances in functional and structural MR image analysis and implementation as FSL. Neuroimage, 23(Suppl. 1), S208-S219.

Tsao, D. Y., Schweers, N., Moeller, S., \& Freiwald, W. A. (2008). Patches of face-selective cortex in the macaque frontal lobe. Nature Neuroscience, 11, 877-879.

Tsukiura, T., \& Cabeza, R. (2011). Remembering beauty: Roles of orbitofrontal and hippocampal regions in successful memory encoding of attractive faces. Neuroimage, 54 , 653-660.

Vartanian, O., Navarrete, G., Chatterjee, A., Fich, L. B., Leder, H., Modroño, C., et al. (2013). Impact of contour on aesthetic judgments and approach-avoidance decisions in architecture. Proceedings of the National Academy of Sciences, U.S.A., 110(Suppl. 2), 10446-10453.

Von Der Heide, R. J., Skipper, L. M., \& Olson, I. R. (2013). Anterior temporal face patches: A meta-analysis and empirical study. Frontiers in Human Neuroscience, 7, 17.

Watson, K. K., \& Platt, M. L. (2012). Social signals in primate orbitofrontal cortex. Current Biology, 22, 2268-2273.

White, M., Smith, A., Humphryes, K., Pahl, S., Snelling, D., \& Depledge, M. (2010). Blue space: The importance of water for preference, affect, and restorativeness ratings of natural and built scenes. Journal of Environmental Psychology, 30, 482-493.

Winston, J. S., O'Doherty, J., Kilner, J. M., Perrett, D. I., \& Dolan, R. J. (2007). Brain systems for assessing facial attractiveness. Neuropsychologia, 45, 195-206.

Yue, X., Vessel, E. A., \& Biederman, I. (2007). The neural basis of scene preferences. NeuroReport, 18, 525-529. 